DOI: $\underline{\text { https://doi.org/10.24297/jam.v17i0.8413 }}$

\title{
Division and Combination in Linear Algebra
}

\author{
Rui Chen", Liang Fang ${ }^{b}$ \\ College of Mathematics and Statistics, Taishan University, 271000, Tai'an, China \\ bfangliang3@163.com. achenruimengting@163.com
}

\begin{abstract}
.
In this paper, the relationship between matrix operation, linear equations, linear representation of vector groups and linear correlation is discussed, and the idea of division and combination in linear algebra is discussed to help learners understand the connections between various knowledge points of linear algebra from multiple angles, deep levels, and high dimensions.
\end{abstract}

Keywords: Matrix Operations, Linear Equations, Linear Representation, Linear Correlation, Divide And Combine

\section{Introduction}

From the definition of the matrix it seems to be impossible to see its relationship with the linear system of equations and the linear representation and linear correlation of vectors. It is not difficult to find out that they are inextricably linked with each other. The operation of the matrix, especially the multiplication operation, changes the representation of the linear equations. The introduction of the rank of the matrix expounds the classification of the solutions of the linear equations. Using the rank of the coefficient Matrix, it is easy to determine the solution of the linear equations.

Although the definition of the linear correlation of vector groups is a basic method for determining the linear correlation of vector groups, it is sometimes inconvenient. Constructing an appropriate homogeneous linear system of equations can determine the linear correlation of the vector system by whether there is a solution to the linear system of equations [1]. At the same time, the solution of the homogeneous linear system of equations can be determined based on the rank of the coefficient matrix. Therefore, we only need to use the vector group as a column matrix to determine the linear correlation of the vector group by finding its rank.

\section{The idea of division and union}

\subsection{Linear equations and matrix multiplication}

1. Linear equations

$$
\left\{\begin{array}{c}
a_{11} x_{1}+\cdots+a_{1 n} x_{n}=b_{1} \\
\cdots \\
a_{m 1} x_{1}+\cdots+a_{m n} x_{n}=b_{m}
\end{array}\right.
$$

Applying matrix multiplication,(1) can be expressed as 


$$
\left\{\begin{array}{c}
\left(a_{11}, \cdots a_{1 n}\right)\left(\begin{array}{c}
x_{1} \\
\vdots \\
x_{n}
\end{array}\right)=b_{1} \\
\cdots \\
\left(a_{m 1}, \cdots a_{m n}\right)\left(\begin{array}{c}
x_{1} \\
\vdots \\
x_{n}
\end{array}\right)=b_{m}
\end{array}\right.
$$

The matrix $\left(\begin{array}{ccc}a_{11} & \cdots & a_{1 n} \\ \vdots & & \vdots \\ a_{m 1} & \cdots & a_{m n}\end{array}\right)$ is divided into rows from the first line to the last line, note as

$\left(a_{11}, \cdots a_{1 n}\right), \cdots,\left(a_{m 1}, \cdots a_{m n}\right)$.

(2) get together, get

$$
\left(\begin{array}{ccc}
a_{11} & \cdots & a_{1 n} \\
\vdots & & \vdots \\
a_{m 1} & \cdots & a_{m n}
\end{array}\right)\left(\begin{array}{c}
x_{1} \\
\vdots \\
x_{n}
\end{array}\right)=\left(\begin{array}{c}
b_{1} \\
\vdots \\
b_{m}
\end{array}\right)
$$

This is the matrix representation of the system of linear equations [1].

2. Matrix multiplication

$$
A B=C
$$

where $A=\left(a_{i j}\right)_{m \times n}, B=\left(b_{i j}\right)_{n \times s}, C=\left(c_{i j}\right)_{m \times s}$.

The matrix $B$ is grouped by column,

$$
B=\left(\beta_{1}, \cdots, \beta_{S}\right)
$$

And the matrix $C$ is grouped by column,

$$
C=\left(\gamma_{1}, \cdots, \gamma_{S}\right)
$$

(4) Separate and become 


$$
\left\{\begin{array}{c}
A \beta_{1}=\gamma_{1} \\
\cdots \\
A \beta_{s}=\gamma_{s}
\end{array}\right.
$$

There are $s$ linear equations inside.

Obviously, from(2) to(3) is integration, from(4) to(5) is split.

Since the linear equations can be represented in matrix form, the matrix relations $A_{m \times n} B_{n \times s}=C_{m \times s}$, from the point of view of points, consider $i$-column of $B$ as a solution to the system of linear equations $A_{m \times n} X=C_{i}$, where $C_{i}$ is column $i$ of $C$.Especially when $C_{m \times s}=0$, it can be associated with homogeneous linear equations. The sufficient necessary conditions for a solution to a linear system of equations are available: the sufficient necessary condition for a solution of matrix equations $A_{m \times n} X_{n \times s}=C_{m \times s}$ is $R(A)=R(A, C)$. The matrix and linear equations can sometimes be further linked to eigenvalues and eigenvectors.

\subsection{Linear representation and matrix multiplication of vector groups}

1. Column vector group $\beta_{1}, \cdots \beta_{n}$ can be linearly represented by column vector group $\alpha_{1}, \cdots \alpha_{m}$, that there is a set of numbers $k_{1 j}, \cdots k_{m j}$, we have

$$
\beta_{j}=k_{1 j} \alpha_{1}+\cdots+k_{m j} \alpha_{m}, j=1, \cdots, n
$$

Matrix multiplication can be expressed as

$$
\beta_{j}=\left(\alpha_{1}, \cdots, \alpha_{m}\right)\left(\begin{array}{c}
k_{1 j} \\
\vdots \\
k_{m j}
\end{array}\right), j=1, \cdots, n .
$$

With the idea of integration, we have

$$
\left(\beta_{1}, \cdots \beta_{n}\right)=\left(\alpha_{1}, \cdots \alpha_{m}\right)\left(\begin{array}{ccc}
k_{11} & \cdots & k_{1 n} \\
\vdots & & \vdots \\
k_{m 1} & \cdots & k_{m n}
\end{array}\right)
$$

Expressed as matrix multiplication, it is

$$
B=A K ，
$$


where $B=\left(\beta_{1}, \cdots \beta_{n}\right), A=\left(\alpha_{1}, \cdots \alpha_{m}\right), K=\left(\begin{array}{ccc}k_{11} & \cdots & k_{1 n} \\ \vdots & & \vdots \\ k_{m 1} & \cdots & k_{m n}\end{array}\right)$, the matrix $K$ is a matrix of coefficients represented by this linear representation [1].

2. Line vector groups $\beta_{1}{ }^{T}, \cdots \beta_{n}{ }^{T}$ can be linearly represented by line vector groups $\alpha_{1}{ }^{T}, \cdots \alpha_{m}{ }^{T}$, that there is a set of numbers $k_{j 1}, \cdots k_{j m}$, we have

$$
\beta_{j}^{T}=k_{j 1} \alpha_{1}^{T}+\cdots+k_{j m} \alpha_{m}^{T}, j=1, \cdots, n
$$

Matrix multiplication can be expressed as

$$
\beta_{j}^{T}=\left(k_{j 1}, \cdots, k_{j m}\right)\left(\begin{array}{c}
\alpha_{1}^{T} \\
\vdots \\
\alpha_{m}^{T}
\end{array}\right), j=1, \cdots, n .
$$

With the idea of integration, we have

$$
\left(\beta_{1}^{T}, \cdots \beta_{n}^{T}\right)=\left(\begin{array}{ccc}
k_{11} & \cdots & k_{1 m} \\
\vdots & & \vdots \\
k_{n 1} & \cdots & k_{n m}
\end{array}\right)\left(\begin{array}{c}
\alpha_{1}^{T} \\
\vdots \\
\alpha_{m}^{T}
\end{array}\right)
$$

Expressed as matrix multiplication, it is

$$
B=K A \text {, }
$$

where $B=\left(\beta_{1}^{T}, \cdots \beta_{n}^{T}\right), A=\left(\alpha_{1}^{T}, \cdots \alpha_{m}{ }^{T}\right), K=\left(\begin{array}{ccc}k_{11} & \cdots & k_{1 m} \\ \vdots & & \vdots \\ k_{n 1} & \cdots & k_{n m}\end{array}\right)$ the matrix $K$ is a matrix of coefficients represented by this linear representation.

From the right side of equation (6), it can be seen that the linearity of the column vector is shown, and its combination coefficient is written in the matrix on the right; From the right side of the equation (8), the linear representation of the row vector is written in the matrix on the left. Therefore, it can be summarized as: "Linear table, combination coefficient, row left column right."

The (7) and (9) formulas obtained by (6) and (8) aggregation respectively have the same conclusion.

The matrix form of the linear table of the row vector group and column vector group has a combination coefficient of left and right. From the point of view of splitting, from the above analysis, if there is a relationship 
between matrices: $T_{m \times n}=U_{m \times s} V_{s \times n}$, then the column vector group of the matrix $T$ can be shown linearly by the column vector group of the matrix $U$; The row vector group of matrix $T$ can be shown linearly by the row vector group of matrix $V$ [2]. In short, the idea of aggregation and the relationship of vector groups can be expressed by Matrix and its operations.

\section{Application Examples}

Example 1 Assume that $A_{m \times n} B_{n \times s}=0, R(A)+R(B) \leq n$. can be proved [2].

Analysis: with the idea of integration, the matrix relations are associated with homogeneous linear equations, and then the theory of equations can be used.

Proof Divide $B$ into columns as, $B=\left(\beta_{1}, \cdots, \beta_{s}\right)$, and $A_{m \times n} B_{n \times s}=0$, we have

$$
A B=A\left(\beta_{1}, \cdots, \beta_{s}\right)=\left(A \beta_{1}, \cdots, A \beta_{s}\right)=(0, \cdots, 0),
$$

Namely,

$$
A \beta_{i}=0, i=1, \cdots, s .
$$

Namely, the column vectors of $B$ are solutions to homogeneous linear equations $A X=0$.

Set $S$ as the solution set of homogeneous linear equations $A X=0$, then

$$
R\left(\beta_{1}, \cdots, \beta_{s}\right) \leq R(S)=n-R(A),
$$

Namely,

$$
R(A)+R(B) \leq n
$$

Example 2 Third order matrix $A$ and three-dimensional column vector $\beta$, making $\beta, A \beta, A^{2} \beta$ linear independent, and $A^{3} \beta=5 A \beta-3 A^{2} \beta$, if $P=\left(\beta, A \beta, A^{2} \beta\right)$, find the third-order matrix so that $A=P B P^{-1}$.

Analysis: Transform $A=P B P^{-1}$ into $A P=P B$, use the idea of splitting, transform the relationship between matrices into the relationship between vector groups, and then use the idea of integration to express it as a whole. Therefore, it is reduced to the form of a matrix. That is, the required column $B$ is the combined coefficient when the column $A P$ uses the linear table of the column vector $P$ [3]. 
Proof For $P=\left(\beta, A \beta, A^{2} \beta\right)$, we have

$$
\begin{aligned}
& A P=A\left(\beta, A \beta, A^{2} \beta\right) \\
& =\left(A \beta, A^{2} \beta, A^{3} \beta\right) \\
& =\left(A \beta, A^{2} \beta, 5 A \beta-3 A^{2} \beta\right) \\
& =\left(\beta, A \beta, A^{2} \beta\right)\left(\begin{array}{ccc}
0 & 0 & 0 \\
1 & 0 & 5 \\
0 & 1 & -3
\end{array}\right)=P B
\end{aligned}
$$

where $B=\left(\begin{array}{ccc}0 & 0 & 0 \\ 1 & 0 & 5 \\ 0 & 1 & -3\end{array}\right)$.

Since $\beta, A \beta, A^{2} \beta$ is linear independent, so $P$ is reversible, and $A=P B P^{-1}$. Then $B$ is what is required.

Example 3 Set a linear system of equations $\left\{\begin{array}{c}a_{11} x_{1}+\cdots+a_{1 m} x_{m}=b_{1} \\ \cdots \\ a_{m 1} x_{1}+\cdots+a_{m m} x_{m}=b_{m}\end{array}\left(^{*}\right)\right.$, If for any $b_{1}, \cdots, b_{m},\left(^{*}\right)$ is solved, then the determinant of the coefficient $\operatorname{det}\left(a_{i j}\right) \neq 0$ of $\left({ }^{*}\right)[4]$.

Analysis: The equations are represented in matrix form, and special ones are taken arbitrarily [5].

Proof From what is known, for $\varepsilon_{1}=(1,0, \cdots, 0)^{T}, \cdots, \varepsilon_{m}=(0, \cdots 0,1)^{T},\left(^{\star}\right)$ has solutions,

set the solution is

$$
\gamma_{i}, i=1, \cdots, m
$$

So

$$
A \gamma_{i}=\varepsilon_{i}, i=1, \cdots, m \text {, }
$$

where $A=\left(a_{i j}\right)_{n}$ so

$$
A\left(\gamma_{1}, \cdots, \gamma_{m}\right)=\left(\varepsilon_{1}, \cdots, \varepsilon_{m}\right)=E_{m} .
$$


Where $E_{m}$ is unit matrix, so $\operatorname{det}(A B)=\operatorname{det}(A) \operatorname{det}(B)=\operatorname{det}(E)=1$. So we have $\operatorname{det}\left(a_{i j}\right) \neq 0$.

Example 4 Assume that $A_{m \times s} B_{s \times m}=E_{m}$, where $m<s$, so column vector group of $A_{m \times s}$ is linearly related.

Analysis: By linking the vector groups by Matrix blocks, we can see that $A_{m \times s}$ and row vector groups of $E_{m}$ are equivalent [6].

Proof For $A_{m \times s} B_{s \times m}=E_{m}$, column vector groups of unit matrices $E_{m}$, namely the $m$-dimensional single row vector group can be linearly represented by the column vector group of $A_{m \times s}$, Obviously, the column vector group of $A_{m \times s}$ can be linearly represented by a $m$-dimensional single row vector group, so they are equivalent, rank is $m$, so $R(A)=m$, and $m<s$, we have the column vector of $A_{m \times s}$ is a linear correlation vector group.

\section{Summary}

Using the dialectical idea of division and combination, the linear representation and linear correlation of matrix and linear equations and vector groups can be converted to each other, so that the knowledge that can be used can be more abundant; Using the dialectical idea of division and combination, the problems to be solved are shown from the microscopic or macroscopic level, so that we can observe it from the microscopic or macroscopic level and think about it, which broadens our thinking about solving the problem; By using the dialectical thought of division and combination, we can activate the breadth and depth of our mathematical thinking and improve the quality of mathematical thinking [7].

\section{Acknowledgment}

The work is supported by Teaching Reform and Research Project of Taishan University in 2018: The Theory of Probability Combining Real Life Application and Computer Simulation and the Teaching Reform of Mathematical Statistics Course, and Tai'an Science and Technology Development Project: Probability Theory and Applied Research of Mathematical Statistics and Practice of Innovative Teaching Reform (2018ZC0287), and Project of Natural Science Foundation of Shandong province (ZR2016AM06).

\section{References}

1. Department of Mathematics, Tongji University. Linear algebra. Fourth Edition. Beijing: Higher Education Press, 2003,pp.46-110.

2. X.-P. Yang. In linear algebra, the idea of "gathering zero for integration and making the whole into zero".. Journal of Southwest Normal University(Natural Science Edition), 2009, 34(05), pp. 235-239.

3. Y.-L. Li, Y.-T Yuan. gently test high scores(linear algebra * probability theory and mathematical statistics)- 
analysis of the classification of questions over the years. Beijing: State Administration College Press, 2003,pp.125.

4. Department of Mathematics, Peking University. Advanced algebra. Third edition. Beijing: Higher Education Press, 2003, pp.156.

5. X.-P. Yang. Dialectical thought methods in higher algebra. Journal of Southwest Normal University(Natural Science Edition), 1998, 23(6), pp.730-734.

6. H.-X.Zhu, D.-Q.Zhu. Problem Based Classroom Teaching Evaluation Standard. Journal of Southwest University(Natural Science Edition), 2007, 29(8), pp.168-171.

7. J.-W.Chen. Distribution of matrix eigenvalues.. Journal of Southwest University(Natural Science Edition), 2007, 29(11), pp.46-47. 